\title{
Furfural Formation from Corn Cobs in a One-Pot Method Catalyzed by ZSM-5
}

\author{
Jian $\mathrm{Du}^{1,2}$, Hongling Gao ${ }^{1,2}$, Jing Guan ${ }^{1}$, Guang $\mathrm{Yu}^{1}$, Yuedong Zhang ${ }^{1}$,Haisong \\ Wang ${ }^{1,3 *}$ and Xindong $\mathrm{Mu}^{1 *}$
}

1: Key Laboratory of Biofuels, Qingdao Institute of Bioenergy and Bioprocess Technology, Chinese Academy of Sciences, Qingdao, China, 266101;

2: University of Chinese Academy of Sciences, Beijing, China, 100049;

3: Tianjin Key Laboratory of Pulp \& Paper, Tianjin University of Science \& Technology, Tianjin, China, 300457;

E-mail: wanghs@qibebt.ac.cn; muxd@qibebt.ac.cn

Keywords: Hemicellulose; Xylose; Furfural; Organic solvent; Zeolite

Abstract. This study designed a green one-pot method for furfural production from corn cobs that were heterogeneously catalyzed by recoverable solid-acid ZSM-5. The highest furfural yield of $78.5 \%$ was obtained under the following conditions: $473 \mathrm{~K}, 30 \% \mathrm{ZSM}-5,15 \mathrm{~mL}$ of toluene, and 500 $\mathrm{mM}$ of $\mathrm{NaCl}$ for $2 \mathrm{~h}$. Catalyst recycling study showed that ZSM-5 could be repeatedly reused with high catalytic activity. The furfural yield was still up to $64.0 \%$ even when reused five times. The results showed that corn cobs are a suitable feedstock to produce furfural catalyzed by ZSM-5. This study not only provides basic data for the furaldehyde industry, but also offers an environmentally friendly way to make full use of crops.

\section{Introduction}

Biomass is an efficient source for the production of chemicals, biofuels, and energy and has attracted lot of attention[1]. Lignocellulose, primarily composed of cellulose, hemicellulose, and lignin, is a kind of sustainable and abundant material produced via photosynthesis. About billions of tons of corn cobs are produced annually in the world, and have a great potential to be one of the main feedstocks in the production of furfural[2].

Furfural is an important intermediate chemical from which can be derived many chemical products, such as furfural alcohol, acetyl furan, furoic acid, methyl furan, and THF[3]. Usually, the content of hemicellulose in corn cobs is approximately $29.98 \pm 3.60 \%$. Thus, corn cobs can be used to produce furfural. Xylose dehydration toward furfural has been studied for several decades using mineral acids $\mathrm{HCl}[4], \mathrm{H}_{2} \mathrm{SO}_{4}$ [5], and $\mathrm{H}_{3} \mathrm{PO}_{4}$ [6] as catalysts. However, there is no record of producing furfural from corn cobs in one pot catalyzed by solid acid ZSM-5.

In addition, rapid and continuous removal of furfural from the reaction solution was urgently demanded to obtain a high yield of furfural using organic solvents. Many organic solvents, such as THF[7], cyclopentyl methyl ether (CPME) [8], toluene[9], DMSO[10], butanol and MIBK, have been used. Besides, metallic oxides and chlorides have been reported to increase furfural production. $\mathrm{NaCl}[9], \mathrm{ZnCl}_{2}, \mathrm{AlCl}_{3}$, and $\mathrm{FeCl}_{3}$ have all been studied recently. In this paper, $\mathrm{NaCl}$ was chosen to be added in the reaction mixture and various concentrations were studied to determine the best concentration.

This study aims to produce furfural in one pot from corn cobs in a green method using industrial catalyst ZSM-5 zeolite and to solve the problems of corrosion, pollution, and inefficiency during the traditional production process. In addition, a catalyst recycling study was carried out.

\section{Experimental}

Materials. Corn cobs were supplied by a farm from Pingdu (China) and it contains 30.5wt\% xylose, $35.9 \mathrm{wt} \%$ glucose. All the reagents used in this experiment were of analytical grade. 
Hydrolysis Reaction. All experiments were conducted in one pot using the method of a previous study[9]. Reaction temperature, amount of ZSM-5 and reaction time was studied.

Product Analysis. The furfural in aqueous phase and organic phase was detected by high performance liquid chromatography (HPLC) with a UV detector and evaporative light and gas chromatography(GC), respectively.

\section{Results and Discussion}

\section{Optimization of Furfural Production from Corn Cobs Catalyzed by ZSM-5}
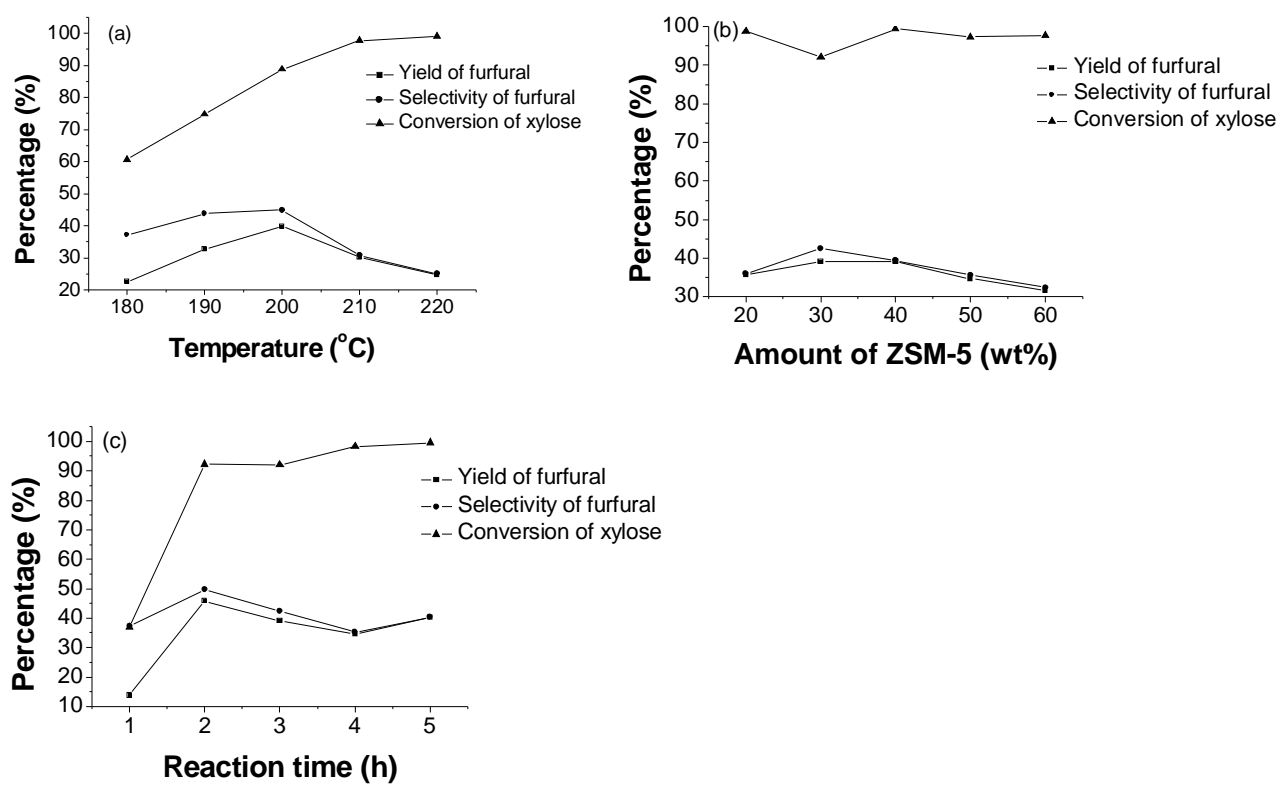

Fig. 1. Furfural yield, selectivity, and xylose conversion under various reaction conditions

Effect of reaction temperature on furfural formation. Different reaction temperatures on the hydrolysis of xylose into furfural was carried out. The results are presented in Fig. 1(a). Obviously, a quicker increase in furfural yield with the temperature rise at the beginning and the maximum furfural yield of $45.9 \%$ could be achieved after $2 \mathrm{~h}$ at $473 \mathrm{~K}$. The conversion of xylose increased sharply as the temperature increased, from $37.0 \%$ to $99.6 \%$. However, furfural yield and selectivity decreased when the temperature was over $473 \mathrm{~K}$. This is probably due to the side reactions of degradation or condensation between furfural and reaction intermediates from xylose at high temperatures[11]. Moreover, furfural is highly unstable and degrades more quickly at high temperatures.

Effect of the amount of ZSM-5 on furfural formation. To study the effect of the amount of ZSM-5 on furfural production, the ratio of solid acid catalyst to corn cobs was varied from $20 \%$ to $60 \%$. The results are shown in Fig. 1(b). The results showed that the yield of furfural increased from $35.6 \%$ to $39.1 \%$ as the solid acid catalyst dosage increased from $20 \%$ to $30 \%$ and the maximum furfural yield of $39.1 \%$ could be obtained after $2 \mathrm{~h}$ at $473 \mathrm{~K}$. The conversion of xylose was close to $100 \%$. However, further increasing the catalyst loading decreased the furfural yield. The reason might be that excessive catalyst enhanced the secondary reactions, such as xylose dehydration to other aldehydes (e.g., formaldehyde) and degradation of furfural, which resulted in undesired by-products[12]. Therefore, the appropriate catalyst dosage could lead to higher furfural yield.

Effect of residence time on furfural formation. To study the influence of residence time on furfural formation, residence times of 1 to $5 \mathrm{~h}$ were employed while keeping other conditions constant. As shown in Fig. 1(c), the conversion of xylose and the furfural yield increased as the residence time increased (with increases from $37.0 \%$ to $92.2 \%$ and $13.8 \%$ to $45.9 \%$, respectively). The maximum furfural yield was achieved at $473 \mathrm{~K}$ for $2 \mathrm{~h}$. Moreover, after reaching the maximum, the furfural yield and its selectivity decreased with further increasing reaction time. This may have occurred because the long reaction time promoted yield-loss reactions and led to the formation of 
soluble degradation products or black insoluble solids[13]. O'Neill et al.[14] revealed this phenomenon in a previous study.

\section{Effect of Organic Solvents on Furfural Formation}
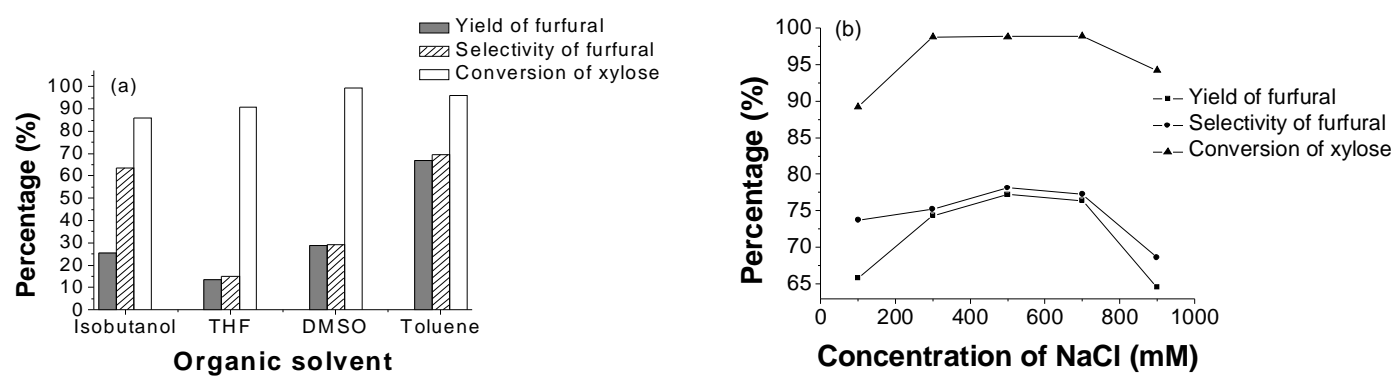

Fig. 2. Effect of (a) organic solvent and (b) $\mathrm{NaCl}$ concentration on yield of furfural, selectivity of furfural, and conversion of xylose

Four organic solvents, i.e., isobutanol, toluene, THF, and DMSO, were utilized as extracting agents. Figure 2(a) shows that the maximum furfural yield of $66.8 \%$ and xylose conversion of $96.1 \%$ could be obtained in a toluene/water system. Furthermore, a furfural yield of $25.4 \%$ could be achieved in an isobutanol/water system. The THF/water system and DMSO/water system resulted in furfural yields of $13.4 \%$ and $28.9 \%$, respectively. Xylose conversion decreased in the following order: DMSO $>$ Toluene $>$ THF $>$ Isobutanol. Previous studies reported that toluene was the preferred solvent as an extracting agent[15]. The same results are shown in our experiments. Once furfural was formed, it could sequentially react in the aqueous phase with the presence of catalysts. However, furfural can be extracted into the organic phase immediately to prevent further secondary reactions because the catalyst only exists in the aqueous phase. Therefore, toluene was the best choice.

\section{Effect of $\mathrm{NaCl}$ on Furfural Formation}

In a previous study, it was found that $\mathrm{NaCl}$ makes the largest contribution to furfural yield, compared to $\mathrm{FeCl}_{3}, \mathrm{CaCl}_{2}$, and $\mathrm{KCl}$. It can be thus used as a promoter in furfural formation. Therefore, various concentrations of $\mathrm{NaCl}$, from 100 to $900 \mathrm{mM}$, were investigated; the results are shown in Fig. 2(b). The furfural yield increased as the concentration of $\mathrm{NaCl}$ increased, and the maximum furfural yield of $77.2 \%$ was obtained at $500 \mathrm{mM}$. This could be attributed to the salting-out effect of $\mathrm{NaCl}$, which decreased the solubility of furfural in water, forcing furfural to transfer to the organic phase. The mechanism of furfural formation in the presence of chloride ions has been reported in previous work[16]. On the other hand, halide ions play an important role in the reaction. Chloride ions have an effect on the hydrolysis of hemicellulose and cyclodehydration of xylose to obtain furfural. Previous studies also have shown that chloride ions reinforce the degradation of xylose and xylan. When the $\mathrm{NaCl}$ concentration was over $500 \mathrm{mM}$, the yield of furfural and conversion of xylose decreased slowly until the concentration reached $700 \mathrm{mM}$, then decreased remarkably above $700 \mathrm{mM}$. According to the Debye-Huckel theory, the ion's activity decreased while the salt concentration increased; consequently, the acid activity decreased, leading to a lower furfural yield and xylose conversion.

\section{Catalyst Recycling Study}

In order to achieve the green and economy goal, a catalyst recycling study was carried out. The catalyst from the reaction mixture was simply washed with diluted acid and water after each use, then calcined for the next reaction. As shown in Fig. 3, the furfural yield obtained from the first run was the highest, although the furfural yields obtained using the recycled catalyst were all similar. A furfural yield of $64.0 \%$ was achieved in the fifth recycle run, a decrease of approximately $11 \%$ compared to the first run. This indicated that the catalyst activity was reduced. ZSM-5 has weak acidic sites and strong acidic sites. After the first run, the stronger acidic sites were damaged and the weaker acidic sites remained stable during the reaction process. 

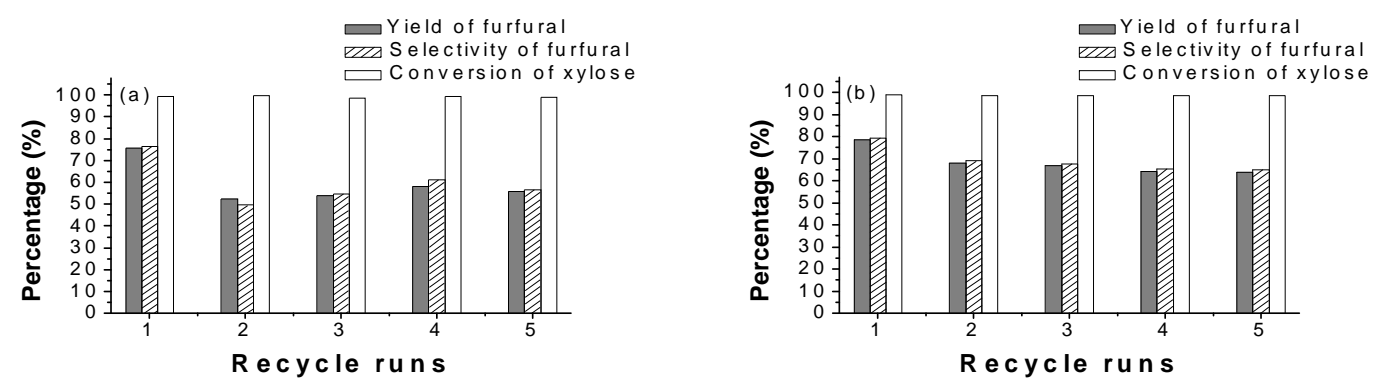

Fig. 3. Recycle runs for catalyst: (a) catalyst was washed with distilled water; (b) catalyst was washed with dilute acid

\section{Conclusions}

Furfural yield can be improved by changing the reaction conditions (reaction temperature, time, amount of catalyst, solid-liquor ratio, type of organic solvent, and salt). The optimal reaction conditions are $473 \mathrm{~K}, 30 \%$ solid acid, corn cob to aqueous solution ratio of 1:10, 20 to 40 mesh, 15 $\mathrm{mL}$ of toluene, $500 \mathrm{mM}$ of $\mathrm{NaCl}$, and heating for $2 \mathrm{~h}$. Under optimized reaction conditions, $78.5 \%$ furfural yield was obtained.

The catalyst recycling study showed that ZSM-5 could be recycled more than five times; using the above conditions, the furfural yield was still up to $64.0 \%$.

Diluted acid can improve the activity of the catalyst. The yield of furfural increased from 55\% to $64 \%$ when the catalyst was washed with diluted acid during the catalyst recycling study.

\section{Acknowledgements}

We are grateful for the support of the Natural Science Foundation of China (No. 21206184 and 31370582), the National High Technology Research and Development Program ("863" program) of China (No. 2012AA022301), and the Project Supported by the Foundation (No. 201304) of Tianjin Key Laboratory of Pulp \& Paper (Tianjin University of Science \& Technology), P. R. China.

\section{References}

[1] P.L. Dhepe, R. Sahu, A solid-acid-based process for the conversion of hemicellulose, Green Chemistry, 12 (2010) 2153-2156.

[2] H.D. Mansilla, J. Baeza, S. Urzua, G. Maturana, J. Villasenor, N. Duran, Acid-catalysed hydrolysis of rice hull: Evaluation of furfural production, Bioresource Technology, 66 (1998) 189-193.

[3] J.M.R. Gallo, D.M. Alonso, M.A. Mellmer, J.H. Yeap, H.C. Wong, J.A. Dumesic, Production of Furfural from Lignocellulosic Biomass Using Beta Zeolite and Biomass-Derived Solvent, Topics in Catalysis, 56 (2013) 1775-1781.

[4] O. Yemiş, G. Mazza, Acid-catalyzed conversion of xylose, xylan and straw into furfural by microwave-assisted reaction, Bioresource Technology, 102 (2011) 7371-7378.

[5] H.S. Bamufleh, Y.A. Alhamed, M.A. Daous, Furfural from midribs of date-palm trees by sulfuric acid hydrolysis, Industrial Crops and Products, 42 (2013) 421-428.

[6] S.K. Uppal, R. Kaur, Hemicellulosic Furfural Production from Sugarcane Bagasse Using Different Acids, Sugar Tech, 13 (2011) 166-169.

[7] C.M. Cai, T.Y. Zhang, R. Kumar, C.E. Wyman, THF co-solvent enhances hydrocarbon fuel precursor yields from lignocellulosic biomass, Green Chemistry, 15 (2013) 3140-3145.

[8] M.J.C. Molina, R. Mariscal, M. Ojeda, M.L. Granados, Cyclopentyl methyl ether: A green co-solvent for the selective dehydration of lignocellulosic pentoses to furfural, Bioresource Technology, 126 (2012) 321-327. 
[9] H.L. Gao, H.T. Liu, B. Pang, G. Yu, J. Du, Y.D. Zhang, H.S. Wang, X.D. Mu, Production of furfural from waste aqueous hemicellulose solution of hardwood over ZSM-5 zeolite, Bioresource Technology, 172 (2014) 453-456.

[10] S.B. Kim, S.J. You, Y.T. Kim, S. Lee, H. Lee, K. Park, E.D. Park, Dehydration of D-xylose into furfural over H-zeolites, Korean Journal of Chemical Engineering, 28 (2011) 710-716.

[11] B. Danon, L. van der Aa, W. de Jong, Furfural degradation in a dilute acidic and saline solution in the presence of glucose, Carbohydrate Research, 375 (2013) 145-152.

[12] C.G. Rong, X.F. Ding, Y.C. Zhu, Y. Li, L.L. Wang, Y.N. Qu, X.Y. Ma, Z.C. Wang, Production of furfural from xylose at atmospheric pressure by dilute sulfuric acid and inorganic salts, Carbohydrate Research, 350 (2012) 77-80.

[13] J. Zhang, J. Zhuang, L. Lin, S. Liu, Z. Zhang, Conversion of D-xylose into furfural with mesoporous molecular sieve MCM-41 as catalyst and butanol as the extraction phase, Biomass and Bioenergy, 39 (2012) 73-77.

[14] R. O'Neill, M.N. Ahmad, L. Vanoye, F. Aiouache, Kinetics of Aqueous Phase Dehydration of Xylose into Furfural Catalyzed by ZSM-5 Zeolite, Industrial \& Engineering Chemistry Research, 48 (2009) 4300-4306.

[15] R. Sahu, P.L. Dhepe, A One-Pot Method for the Selective Conversion of Hemicellulose from Crop Waste into C5 Sugars and Furfural by Using Solid Acid Catalysts, Chemsuschem, 5 (2012) 751-761.

[16] G. Marcotullio, W. de Jong, Furfural formation from D-xylose: the use of different halides in dilute aqueous acidic solutions allows for exceptionally high yields, Carbohydrate Research, 346 (2011) 1291-1293. 